Esta obra está bajo una Licencia Creative Commons

Atribución-NoComercial-Compartirlgual 4.0 Internacional

La imprudencia de Pandora: enfoques transversales en la formación universitaria

Carlos Zavaro Pérez

Trayectorias Universitarias, 7 (13), e081, 2021

ISSN 2469-0090 | https://doi.org/10.24215/24690090e081

https://revistas.unlp.edu.ar/TrayectoriasUniversitarias

Universidad Nacional de La Plata

La Plata | Buenos Aires | Argentina

\title{
La imprudencia de Pandora: enfoques transversales en la formación universitaria
}

Pandora's imprudence: transversal approaches in university education

\author{
Carlos Zavaro Pérez \\ https://orcid.org/0000-0003-3298-7383 \\ czavaro@fcnym.unlp.edu.ar \\ Facultad de Ciencias Naturales y Museo | \\ Universidad Nacional de La Plata | Argentina
}

\section{RESUMEN}

La formación universitaria está estructurada en planes de estudio que articulan fragmentos del conocimiento aportado por la ciencia. Las relaciones que establecemos con el ambiente y nuestros congéneres son parte de los constructos que han consolidado un modelo socio-económico dominante y expulsivo que emerge como resultado de una crisis de la racionalidad y del humanismo. Superar la escisión del saber instituido e intentar transversalizar la enseñanza, integrando saberes disciplinares e incorporando otras temáticas como la perspectiva de género y los derechos humanos con énfasis en el derecho de las minorías, la convivencia democrática, el énfasis en la memoria colectiva y la mirada socioambiental, requiere de un formato pedagógico que proponga el debate, el revisionismo y el pensamiento crítico. Las prácticas integrales en ese marco pueden constituir un espacio relevante en relación a la formación de los futuros profesionales.

\section{ABSTRACT}

University education is structured in curricula. Courses articulate fragments of scientific knowledge. The relationships we maintain with the environment and with other human beings are part of the constructs that have consolidated a dominant and expulsive socioeconomic model. This situation emerges as a crisis of rationality and humanism. In this context, it is important to overcome the division of knowledge by integrating disciplinary knowledge within the framework of situated learning and incorporating other topics such as the gender perspective and human rights with emphasis on the rights of minorities, democratic coexistence, emphasis on collective

PALABRAS CLAVE

prácticas integrales, democracia, género, ambiente, humanística.

\section{KEY WORDS}

integral practices, democracy, gender, environment humanistics. 


\section{INTRODUCCIÓN}

El desafío de Prometeo, restituyéndole el fuego a los humanos, desató la venganza de Zeus materializada en la imagen de una mujer moldeada en arcilla, dotada de gran belleza y sensualidad, de gran capacidad para manipular y mentir, y de una asombrosa curiosidad. Poco tiempo después de casarse con Epimeteo, hermano de Prometeo, recibió una enigmática caja destinada a su marido con la advertencia de no abrirla jamás. Sin embargo, su imprudencia la llevó a hurgar en su interior, provocando que todos los males contenidos en ella se dispersaran por el mundo.

La leyenda, que se ha instalado en el imaginario de la cultura occidental, ha representado un modo de delegar responsabilidades y una manera de justificar metafóricamente las calamidades que azotan a la humanidad, exculpando al modelo hegemónico de desarrollo y al modo en que éste ha concebido la producción de bienes de consumo y de sentido en torno a éstos.

\section{LA CAUSA DE TODOS LOS MALES}

Es posible que la caja de Pandora haya quedado en la mitología como origen de todos los males. La deshonestidad, la apatía, el egoísmo y la mezquindad, la indiferencia, la soberbia, la manipulación y la arrogancia, la avaricia, la intolerancia, el racismo, la ira, la crueldad, la incapacidad de manifestar empatía, el orgullo y la violencia son algunos de ellos. Pareciera tan solo una exageración, pero no hay dudas 
que la metáfora nos interpela. Basta mirar alrededor para convencernos que vivimos en un entorno colmado de situaciones de discriminación étnica o cultural y de violencias a ciertos colectivos o individuos por su orientación sexual o género. Ese entorno, además, está visiblemente deteriorado como resultado de una lógica profundamente economicista (Gudynas, 2003) y acrítica.

El modelo devela y exhibe la maledicencia contenida en la famosa caja. El sistema y los códices con que vivimos son resultado del modo en que nos percibimos y nos hemos construido como sujetos sociales (Zemelman, 2006), de la manera en que nos relacionamos con nuestros semejantes y con el entorno y, en consecuencia, concebimos y gestionamos los recursos con que nos proveemos. Si consideramos que los minerales, las moléculas, las especies que nos rodean e incluso las personas, constituyen recursos, naturales y humanos, el entorno resulta tan sólo un supermercado. La afirmación, lejos de ser otra metáfora, constituye la foto de un dispositivo de producción de sentidos que fomenta la acumulación de la riqueza (Ávila, 2019) a expensas, incluso, de nuestra posibilidad de subsistencia como especie (Zavaro Pérez, 2018).

\section{LA RACIONALIDAD COMO CLAVE}

Reconocer todo aquello que forma parte del entorno, asignarle valor y significado es posible gracias a la racionalidad que nos caracteriza. Ha sido el sentido común, basado en la inducción, la experiencia empírica devenida en oralidad y el conocimiento legitimado por la ciencia algunos de los modos en que ese conocimiento se ha ido consolidando y transmitiendo por generaciones.

La historia del pensamiento científico es también la historia de la construcción de sentido sobre el mundo. Una historia que ha sido sistematizada, institucionalizada y fragmentada por la mirada cartesiana (Battisti, 2010). Esa historia ha alimentado, a su vez, la historia de las disciplinas científicas, de las bibliotecas que compilan y clasifican los saberes producidos $y$, finalmente, el recorte que conforman las propuestas de los programas de estudio que estructuran la formación universitaria y que, en cierta medida, revelan el modo en que desde esas instituciones se concibe y proyecta un determinado modelo de sociedad.

El conocimiento estructurado en campos disciplinares ha ido delineando los conceptos con los que esa realidad es interpretada provocando una escisión en la comprensión del entorno que ha contribuido a entenderlo como sumatoria de fragmentos configurados por los sesgos que esas tradiciones han introducido. La fragmentación del saber (Pellegrini, 2019) ha contribuido a instalar una mirada disociada de cuánto nos rodea. Ante esta coyuntura me pregunto si es posible una salida. Quizás sea tiempo de repensar la lógica de las currículas universitarias a fin de fomentar una nueva racionalidad. 


\section{El conocimiento estructurado en campos disciplinares ha ido delineando los conceptos con los que esa realidad es inter- pretada provocando una escisión en la comprensión del en- torno que ha contribuido a entenderlo como sumatoria de fragmentos configurados por los sesgos que esas tradiciones han introducido.}

\section{TRANSVERSALIZAR LA ENSEÑANZA}

La posibilidad de conectar conceptos aparentemente disociados en materias diferentes constituye una primera aproximación a la transversalización de la enseñanza (Zavaro Pérez, 2020a). Los nexos explicitados en la currícula como parte del esquema de correlativas en un modelo de enseñanza que va abordando la complejidad en los años superiores resulta insuficiente.

Establecer un eje que permita conectar, a modo de transecta, los conceptos que forman parte de un mismo programa de estudio y, más aún, de programas de materias diferentes, y que sea capaz de recorrer el plan de estudios y trascenderlo, es relevante. No se trata de encastrar, como quien arma un rompecabezas, los conceptos que permiten definir y describir cuanto nos rodea y que han formado parte de la manera de producir conocimiento científico y de pensar la enseñanza desde las disciplinas, sino de incorporar otras perspectivas que sean capaces de atravesar esos contenidos y de resignificarlos.

Si bien algunas temáticas como el género, la estructura de representación democrática, los derechos humanos o la problemática ambiental, constituyen contenidos específicos en algunas áreas de conocimiento, éstos, a su vez, conforman dimensiones que complejizan la manera en que se pueden abordar los saberes específicos en la currícula de cualquier carrera universitaria -e incluso en otros niveles de la educación formal y de la educación no formal-, y que si bien han atravesado la historia de la conformación de las disciplinas, han sido invisibilizados por los mismos procesos de constitución, de institucionalización (Fernández y Torres, 2009; Orozco y Chavarro, 2009) y de naturalización.

No alcanza con la mera mención de estos temas en espacios concebidos a tal fin, sino de permear la formación académica con estas problemáticas. No obstante, este propósito requiere de un formato pedagógico que interpele a las disciplinas, que contextualice la formación, que fomente la discusión y que revalorice la mirada crítica. De esta manera, es necesario apelar a estructuras curriculares más flexibles (Abate y Orellano, 2020) y en ese marco las prácticas integrales, como espacio de aprendizaje colectivo, se configuran como un escenario posible (Zavaro Pérez, 2020b). 
No se trata de encastrar, como quien arma un rompecabezas, los conceptos que permiten definir y describir cuanto nos rodea y que han formado parte de la manera de producir conocimiento científico y de pensar la enseñanza desde las disciplinas, sino de incorporar otras perspectivas que sean capaces de atravesar esos contenidos y de resignificarlos.

\section{LAS PRÁCTICAS INTEGRALES COMO FORMATO}

Las prácticas integrales constituyen per se un espacio desde el que se alienta una lectura compleja de la realidad y el trabajo en base a problemas (Restrepo Gómez, 2005) persigue esa intención. Siendo que los problemas no constituyen ejemplos abstractos de los recortes de las asignaturas, sino situaciones dadas que condicionan el aprendizaje, (Zavaro Pérez, 2020b) es imperioso consensuar una agenda (García Galván y Lindquist Sánchez, 2020) que no sólo esté en consonancia con las prioridades institucionales en relación a la producción de conocimientos y a la inserción de sus egresados en el mercado laboral, sino también con los lineamientos y políticas estatales a mediano y largo plazo y, especialmente, con la agenda de problemas que importa a la comunidad.

Por su parte, los problemas constituyen un insumo que, desde el punto de vista didáctico, posibilitan el debate sobre las causas que los generan, las interacciones emergentes que los modulan y su impacto en el contexto en que ocurren. Competencias como la formulación de hipótesis, la búsqueda rigurosa de información, el uso y la apropiación de diversas metodologías que permitan trabajar de manera autónoma en su diagnóstico y problematización son posibles de desarrollar recorriendo contenidos y resignificándolos en un aprendizaje pensado desde el hacer.

Competencias como la formulación de hipótesis, la búsqueda rigurosa de información, el uso y la apropiación de diversas metodologías que permitan trabajar de manera autónoma en su diagnóstico y problematización son posibles de desarrollar recorriendo contenidos y resignificándolos en un aprendizaje pensado desde el hacer.

Bajo este formato, entonces, es imposible concebir al aprendizaje por fuera del territorio porque es en él donde los problemas toman corporalidad y en el que éstos son descritos también -en primera persona- por la palabra de quienes lo habitan. La complejidad del 
territorio, que es multicausal, conecta muy diversas dimensiones e involucra a diferentes actores que son portadores de miradas muy diferentes. Sin embargo, no se trata de interdisciplinar ¿transdisciplinar? la enseñanza y promover el diálogo entre las teorías y disciplinas desde las que esos problemas puedan analizarse. Tampoco se trata solamente de incentivar el diálogo con la comunidad (vecinas/ os, instituciones y organizaciones sociales) sino de complejizar la comprensión de esos problemas incorporando aquellas dimensiones que develan la trama indisoluble que los caracteriza, una trama que según Abate y Orellano (2020) es controversial y constituye un tejido o red (Nudler, 2009) que es constituyente del tejido social.

\section{DIMENSIONES DEL ANÁLISIS}

A esta altura del análisis, es posible sostener que la formación transversal debería ser superadora del diálogo de saberes, integrando las dimensiones humanísticas y socio-ambientales que emergen y se explicitan cuando el aula es el territorio, los problemas son situaciones concretas y el saber disciplinar es presentado de manera contextualizada.

\section{esta altura del análisis, es posible sostener que la formación transversal debería ser superadora del diálogo de saberes, in- tegrando las dimensiones humanísticas y socio-ambientales que emergen y se explicitan cuando el aula es el territorio, los problemas son situaciones concretas y el saber disciplinar es presentado de manera contextualizada.}

La construcción de sentido en torno al género, por citar ejemplos, ha condicionado históricamente la dinámica de las disciplinas que ha estado influenciada por el patriarcado hegemónico que encuentra espejo también en el mundo científico. Tan es así, que el rol de la mujer ha sido, salvo excepciones, relegado o revindicado tardíamente como parte del proceso de feminización de las universidades (Baro, 2021). El acceso de las mujeres a puestos de decisión en la comunidad académica aún es escaso a pesar de las conquistas de los movimientos feministas. El techo de cristal (Guil Bozal, 2008) y el Ilamado piso pegajoso (Upegui Valencia y Cervera Delgado, 2018) constituyen teorías que contribuyen a visibilizar estas situaciones y que deberían trabajarse como parte de la historia revisada de las disciplinas.

De igual manera la discriminación de ciertos colectivos por sus orientaciones sexuales ha sido un impedimento para el ejercicio de determinadas profesiones y para el modo en que la sociedad ha construido sentido sobre quienes lo integran. Si esto ocurre hacia el interior de la 
academia es de esperar que se proyecte, de igual manera, en la comunidad. En consecuencia, muchos de los problemas que la afectan y que podrían ser parte de la didáctica de la formación universitaria, inevitablemente estarían alcanzados por estas problemáticas. La formación de futuros egresados en relación a los derechos de las personas, tanto en el ejercicio de su profesión como en los vínculos que como sujetos establecen con otros ciudadanos, es y debe ser prioritaria.

También el impacto de los gobiernos de facto ha dejado huella en la sociedad argentina y en la academia. La desaparición de toda una generación, a consecuencia de la dictadura, es un hecho y muchos de las/os jóvenes perseguidos - desaparecidos eran docentes y estudiantes universitarios (Águila, 2020) e incluso alumna/os de los últimos años del nivel secundario. El desarraigo provocado por el exilio de otros tantos y las relaciones que se establecieron hacia el interior de los campos disciplinares en ese contexto ha marcado también la producción de conocimientos en la ciencia nacional.

No es posible pensar la formación sin traslucir y, más aún, poner en debate permanente la impronta que los gobiernos totalitarios dejaron en nuestra sociedad, especialmente en una época en que el avance de la derecha se hace notorio en la región. La memoria colectiva en este sentido es crucial como forma de perpetuar las representaciones en sentido histórico de aquellos hechos que determinaron nuestra identidad como nación y como sociedad (De Amézola, 2011). Ser conscientes del pasado para gestionar el futuro es más que importante en la formación de quienes han de desempeñar roles de conducción en la gestión en el mediano plazo y en ese sentido no basta presentar la historia reciente como parte de un programa de contenidos, sino que ésta debe impregnar toda la lógica de la formación académica.

En ese sentido, el formato de las prácticas integrales suele fomentar el diálogo, tanto entre diferentes saberes como entre las personas, y éste constituye uno de los valores que representa al sistema democrático. Más allá del ejercicio del diálogo y del valor que le es inherente, es importante poner en discusión también la relevancia que tiene la diversidad y los intereses que se sostienen en torno a un determinado problema desde los diferentes colectivos que conforman una comunidad, siendo que los intereses constituyen la base de la disputa de poder en el sistema democrático (Vidiella, 2013). La disputa de intereses y de sentidos es parte de la complejidad que representa cualquier problemática sobre la que se pueda centrar un modelo de enseñanza-aprendizaje de esta naturaleza, y la reflexión en torno a este factor no puede ni debería obviarse. En la resolución de los problemas que atañen a la sociedad, la mirada tecnocrática no es suficiente y la formación, por lo tanto, también debe involucrar una mirada política.

Otra de las dimensiones que debe atravesar la formación de grado es la ambiental. Los problemas que impactan a la sociedad no son sino 
problemas socio-ambientales. Más allá de que el concepto de ambiente es polisémico y ha sido interpretado de muy diversas maneras por la sociedad y las disciplinas científicas, el ambiente puede definirse como el entorno en que acontece la vida y las relaciones que le son inmanentes. De esta manera, la agenda ambiental no se circunscribe a temas particulares como el calentamiento global, la conservación de la biodiversidad o la contaminación, por citar algunos ejemplos.

No existe un ambiente natural prístino que nos excluya. La sociedad representa un sistema de organización que subsiste gracias a todo cuanto nos rodea. El destino del ambiente, por ende, está signado por nuestras decisiones y ellas son resultado de una construcción social de sentido, de un conjunto de normas y de disposiciones que rigen nuestros comportamientos y de toda una trama de interacciones en las que ha primado la visión economicista y extractivista (Gudynas, 2018). Los problemas ambientales, no son otra cosa que problemas sociales resultantes de una crisis de la racionalidad (Leff, 2007).

Superar los enfoques multidisciplinares como estrategia para construir un lenguaje común y una nueva racionalidad es imperativo. Nuestro futuro depende de ello y en ello adquiere relevancia una formación holística y transversal que conecte a las/os estudiantes con la vida y la realidad cotidiana (Morales et al., 2015) y que sea capaz de integrar toda esa complejidad -que es en sí misma indisociable- desde una perspectiva política, revisionista, analítica, dialógica y profundamente crítica.

\section{EN SÍNTESIS,}

El saqueo del ambiente en que vivimos para sostener irreflexivamente un modelo de sociedad que fomenta la distribución desigual de la riqueza, y con ella la vulneración de los derechos de las personas, nos enfrenta ante la inminente necesidad de fomentar un cambio en la racionalidad con la que interpretamos nuestro entorno y nos relacionamos con él y con quienes lo compartimos. El modelo basado en el uso irracional de los recursos naturales, en la imposición de sentidos, en la distribución desigual del capital y de sus emergentes, incluido el capital cultural, y en la marginación de las minorías ha sido naturalizado como si las responsabilidades recayeran en la improvisada decisión de Pandora de liberar todos los males como consecuencia de su curiosidad irreprimible.

Un formato pedagógico y una lógica que permee la formación universitaria integrando estos temas y complejizando su análisis en sentido histórico y crítico representa una mirada novedosa en la concepción de la enseñanza y en la consolidación de los propósitos que ésta persigue: no se trata de capacitar tecnócratas descomprometidos con su contexto, sino de formar sujetos capaces de sentirse parte de la 
comunidad en la que se insertan y en la que se desempeñarán como profesionales. Una formación de estas características, inevitablemente, ha de impactar en el tipo de sociedad que es posible construir, una sociedad para la cual Pandora, y su caja, representaría tan solo un mito.

Un formato pedagógico y una lógica que permee la formación universitaria integrando estos temas y complejizando su análisis en sentido histórico y crítico representa una mirada novedosa en la concepción de la enseñanza y en la consolidación de los propósitos que ésta persigue 


\section{BIBLIOGRAFÍA}

Abate, S. M., y Orellano, V. (2020). Temas transversales en acción. Trayectorias Universitarias,1-13. Disponible en: https://doi.org/10.24215/24690090e033

Águila, G. (2020). La Universidad Nacional de Rosario en dictadura (19761983): depuración "normalización" y reestructuración institucional. PolHis. Revista Bibliográfica del Programa Interuniversitario de Historia Política, 14, 145178. Disponible en: http://polhis.com.ar/index.php/polhis/article/view/89

Ávila, M. B. (2019). Mujer y naturaleza: de los sentidos de la dominación en el capitalismo y en el sistema patriarcal. En: Celiberti, L. (coord). Las bases materiales que sostienen la vida: perspectivas ecofeministas. (pp. 35-46). Montevideo: Imprenta Rojo.

Baro, S. M. (2021). Mujeres y Universidad en Argentina: contextos y desafíos. Revista de la Educación Superior, 50(199), 117-128. Disponible en: https://doi. org/10.36857/resu.2021.199.1803

Battisti, C. A. (2010). O método de análise cartesiano e o seu fundamento. Scientiae studia, 8, 571-596.

Guil Bozal, A. (2008). Mujeres y ciencia: techos de cristal. Eccos Revista Científica, 10(1), 213-232. https://www.redalyc.org/pdf/715/71510111.pdf

De Amézola, G. (2011). La última dictadura militar en la escuela argentina: entre la historia reciente y la memoria colectiva. Revista de Teoria y didactica de las Ciencias Sociales, (17), 29-56. Disponible en: https://www.redalyc.org/ pdf/652/65221619003.pdf

Fernández, M. y Torres, C. (2009). La ciencia como institución social: clásicos y modernos institucionalismos en la sociología de la ciencia. Arbor, 185(738), 663-687. Disponible en: https://doi.org/10.3989/arbor.2009.738n1045

García Galván, R., y Lindquist Sánchez, R. (2020). Hacia una agenda social de las universidades latinoamericanas del siglo XXI: una perspectiva teórica-epistémica y política. Revista de la Educación Superior, 49(194), 89-113. Disponible en: https://doi.org/10.36857/resu.2020.194. 1126

Gudynas, E. (2003). Ecología, economía y ética del desarrollo sostenible. Quito: Abya-Yala.

Gudynas, E. (2018). Extractivismos: el concepto, sus expresiones y sus múltiples violencias. Papeles de relaciones ecosociales y cambio global, 143, 61-70. 
Leff, E. (2007). La complejidad ambiental. Polis. Revista Latinoamericana. 16, 1-10. Disponible en: https://journals.openedition.org/polis/4605

Morales, A., Guevara, E., \& Medina, D. (2015). Universidad, transversalidad y sociedad. Revista Iberoamericana de Educación en Ciencias y Tecnología, 6(1), 1-15. Disponible en: http://www.exactas.unca.edu.ar/riecyt/VOL\%206\%20 NUM\%201/A\%20\%201.pdf

Nudler, O. (2009). Espacios controversiales: hacia un modelo de cambio filosófico y científico. Buenos Aires: Miño y Dávila.

Orozco, L. A., y Chavarrro, D. A. (2010). Robert K. Merton (1910-2003). La ciencia como institución. Revista de Estudios Sociales, 37, 143-162. Disponible en: https://doi.org/10.7440/res37.2010.08

Pellegrini, P. A. (2019). La verdad fragmentada: Conflictos y certezas en el conocimiento. Buenos Aires: Argonauta.

Restrepo Gómez, B. (2005). Aprendizaje basado en problemas (ABP): una innovación didáctica para la enseñanza universitaria. Educación y educadores, 8, 9-20. Disponible en: https://dialnet.unirioja.es/servlet/articulo?codigo=2040741

Upegui Valencia, A. M., y Cervera Delgado, C. (2018). Techo de cristal y suelo pegajoso: estudios de género en la academia. Jóvenes en la ciencia, 4(1), 1844-1848. Disponible en: https://www.jovenesenlaciencia.ugto.mx/index. php/jovenesenlaciencia/ article/view/2886

Vidiella, G. (2013). Democracia:¿ razones o pasiones? Tópicos, 25, 63-80. Disponible en: https://www.redalyc.org/pdf/288/28829922004.pdf

Zavaro Pérez, C. (2018). La diversidad biológica y la evolución como garantía de la sustentabilidad de la vida. Perspectivas. Revista científica de la Universidad de Belgrano, 1(1), 201-218. Disponible en: https://revistas.ub.edu.ar/ index.php/Perspectivas/article/view/20

Zavaro Pérez, C. (2020a). Saberes ambientales y extensión como sustrato de las prácticas integrales. Revista EXT, 12, 1-15. Disponible en: https://revistas.unc.edu.ar/index.php/ext/article/view/ 30566

Zavaro Pérez, C. A. (2020b). Algunos interrogantes sobre integrar las prácticas académicas: Reflexiones y Propuestas. Trayectorias Universitarias, 6, 1-11. Disponible en: https://doi.org/10.24215/24690090e035

Zemelman, H. (2006). Pensar la sociedad y a los sujetos sociales. Revista Colombiana de Educación, 50, 15-33. Disponible en: http://hdl.handle. net/20.500.12209/8923. 